\title{
UNILATERAL GIANT HYDRONEPHROSIS IN ADULT FEMALE: A RARE CASE REPORT
}

Shiping Jain ${ }^{1}$, Sandeep Kumar Jain ${ }^{2}$

\section{HOW TO CITE THIS ARTICLE:}

Shiping Jain, Sandeep Kumar Jain. "Unilateral Giant Hydronephrosis in Adult Female: A Rare Case Report". Journal of Evolution of Medical and Dental Sciences 2014; Vol. 3, Issue 31, July 31; Page: 8692-8696,

DOI: $10.14260 /$ jemds/2014/3108

ABSTRACT: Giant hydronephrosis, which causes symptomless enlargement of the abdomen, should be considered in the differential diagnosis of huge intraabdominal cystic masses. Epidemiologically, giant hydronephrosis is an extremely rare condition in adults, with most cases being discovered in young-to-middle-aged people. We report a case of 50 year old female with a giant hydronephrotic right kidney accompanied by renal insufficiency. In this report the importance of diagnostic modalities like ultrasonography and computed tomography in the diagnosis and differential diagnosis of giant hydronephrosis to other cystic masses is emphasized.

KEYWORDS: Giant hydronephrosis, ultrasonography, computed tomography.

INTRODUCTION: Giant hydronephrosis in adults is an extremely rare clinical condition with few reported cases in the literature. ${ }^{1}$ Giant hydronephrosis (GH) was described by Sterling in 1939 as the presence of more than one litre of urine in the collecting system of the kidney.1,2 Crooks et al. proposed that giant hydronephrosis should be defined as a kidney occupying half the abdomen, which meets or crosses the midline and is at least 5 vertebrae in length. ${ }^{3}$ It is seen more often in males than in females (2.4:1) and more often on the left than on the right side (1.8:1). ${ }^{1}$ GH usually occurs secondarily due to ureteropelvic junction obstruction (UPJ). ${ }^{4}$ We report a case of 50 -year-old women with unilateral giant hydronephrosis and concomitant renal insufficiency.

CASE REPORT: A 50 years old female patient was admitted in our hospital, with complaints of abdominal distension, abdominal pain, and dysuria since 4 months. On clinical examination her blood pressure was 140/84 $\mathrm{mmHg}$ and pulse was 82 regular beats/min. The abdomen was distended with a large palpable non-tender mass in the right side of abdomen which extended beyond the midline and to the right iliac fossa inferiorly (Figure 1). The blood laboratory tests highlighted a hemoglobin of $9.1 \mathrm{~g} / \mathrm{dL}$, creatinine of $2.2 \mathrm{mg} / \mathrm{dL}$, and urea of $52 \mathrm{mg} / \mathrm{dL}$.

The abdominal radiograph (Figure 2) showed a large abdominal mass of water density in the right side of abdomen. A complementary radiological study via abdominal ultrasound (Figure 3) IVP (Figure 4) and CT scan (Figure 5) were performed. Ultrasonography showed a large anechoic cystic mass measuring about $21.5 \times 17.5 \mathrm{~cm}$ in size, which occupied the entire right sided abdominal cavity and extended to epigastric and umbilical region medially. There was evidence of markedly thinned out renal parenchyma seen at peripheral portion of cystic mass.

The cystic mass displaced the abdominal structures to opposite side. The left kidney was normal in size and contour and showed mildly raised echo-texture, significantly dilated renal pelvis $(9 \times 4 \mathrm{~cm})$ and non-dilated ureter. On IVU nephrogram could not be visualized in right renal area even on delayed images, suggestive of nonfunctional right kidney. Left kidney showed dilated pelvicalyceal 
system with non-visualisation of left ureter. Urgent Ultrafast, multislice CT intravenous urography was performed using non-ionic contrast.

CT urography showed hugely enlarged right kidney secondary to severe dilatation of the pelvicalyceal system with resultant thinning of the renal parenchyma. There was delayed opacification of the residual cortex but no excretion of contrast even on the delayed images.

The enlarged kidney was seen extending upto the right lobe of liver superiorly and inferiorly up to the iliac crest. The hydronephrotic kidney displaced bowel loops, aorta and the IVC to the left without significant compression. The ureter of the right kidney was not dilated. The left kidney was normal in size and enhancement. There was dilatation of the left renal pelvis noted. The left ureter was not opacified. However it is normal in calibers. So in our case bilateral UPJO was the most likely cause for hydronephrotic kidneys.

DISCUSSION: Giant hydronephrosis is a condition caused by the accumulation of more than 1,000 $\mathrm{mL}$ in the excretory system of either kidney, as defined by Stirling in 1939.1,2 The first case was published in 1746, and more than 600 cases have been described worldwide to date. It is seen more often in males than in females (2.4:1) and more often on the left than on the right side (1.8:1). ${ }^{1}$ In our case, giant hydronephrotic kidney was reported in female patient on right side.

The clinical symptoms of these patients are not specific but typically involve increased abdominal girth due to the presence of a mass in the flank. Other symptoms were described in the literature, including pain at the flank along with hematuria resulting from trauma in the area 5 .

In literature, UPJO was the most common cause of giant hydronephrosis 33\% (as in our case), followed by stones $20 \%$ and congenital ureteral narrowing. ${ }^{1,6}$ Other causes include ureteropelvic tumors, ${ }^{7}$ trauma, retroperitoneal fibrosis, ${ }^{1}$ obstructive megaureter, ureteric atresia and obstructive ectopic ureter with or without a duplex system. ${ }^{6}$

The first radiological method in GH diagnostics is abdominal ultrasonography. Other useful diagnostic imaging techniques include CT / MRI, abdominal radiography and intravenous urography (IVU). Abdominal radiography is useful for observable radiopaque lithiasis. Intravenous urography showing no excretion in the affected kidney.

In most cases, CT and magnetic resonance (MR) are the methods of choice in the differential diagnosis of GH with other intraabdominal cystic masses, especially if renal parenchyma is partially preserved and functional, with contrast enhancement. Even if contrast enhancement is absent due to the atrophy of the renal parenchyma, GH should be included in the differential diagnosis.

The list of differential diagnosis is wide and includes: ovarian cysts, retroperitoneal hematoma, hepatobiliary cysts, mesenteric and hepatobiliary cysts, pseudomyxoma, renal tumour, retroperitoneal tumors, ascites and splenomegaly. ${ }^{8}$

CONCLUSION: We reported a case of unilateral giant hydronephrosis accompanied by renal insufficiency in a fifty year old female. Giant hydronephrosis should be included in the differential diagnosis of huge intraabdominal cystic masses. USG alongside CT /MRI should be the diagnostic method of choice.

\section{REFERENCES:}

1. Chiang P H, Chen M T, Chou YH. Giant hydronephrosis: Report of 4 cases with review of the literature. J Formosan Med Assoc, 89, 811-817 (1990). 
2. Sterling WC. Massive hydronephrosis complicated by hydroureter. Report of 3 cases. J Urol. 1939; 42: 520-33.

3. Crooks K K, Hendren W H, Pfister R C. Giant hydronephrosis in children. J Pediatr Surg, 14, 844847 (1979).

4. Cain MP, Vanderslice RR, Gibons MD. Uretero-Infundibuloplasty for Giant Hydronephrosis. J The Scientific World. 2004; 1: 438-42

5. W T Yang, C Metreweli. Giant hydronephrosis in adults: the great mimic. Early diagnosis with ultrasound. Postgraduate Medical Journal, vol. 71, no. 837, pp. 409-412, 1995

6. Shah SA, Ranka P, Dodiya S, Jain R, Kadam G. Giant hydronephrosis: What is the ideal treatment? Indian Journal of Urology. 2004; 20 (2): 118.

7. Benchekroun A, Alami M, Ghadouane M, et al. Giant hydronephrosis: two case reports. Ann Urol (Paris). 2003; 37 (2): 61-4.

8. Mountney J, Chapple CR, Johnson AG. Giant hydronephrosis-a diagnostic dilemma. Urol Int. 1998; 61: 121-3.

Figure-1.Photo of abdomen of patient showing marked distension right side.

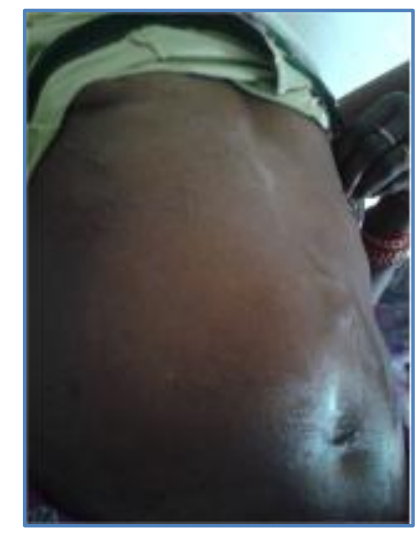

Fig. 1

Figure-2.X ray abdomen showing homogenous opacity occupying most of the right side of abdomen and extending slightly towards the left of midline.

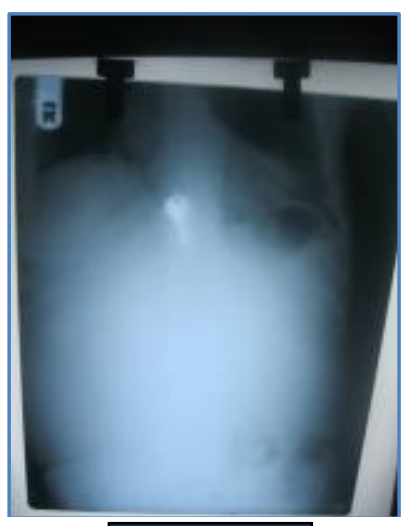

Fig. 2 
Figure-3:- Intravenous urography showing a large homogenous opacity suggestive of dilated pelvicalyceal system.No renal function was seen on right side (non functional right kidney with marked hydronephrotic bag). left kidney show mildy dilated pelvicalyceal system.

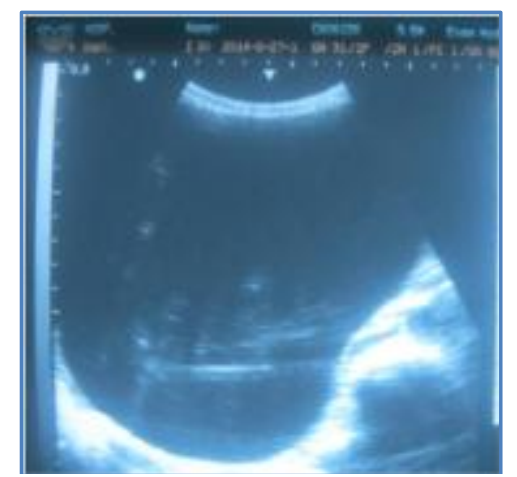

Fig. 3

Figure-4 ultrasound abdomen showing a large anechoeic cystic mass occupying right side of abdomen with intracystic fine level internal echoes.The right kidney not seen separately with marked thinning of the renal cortex.

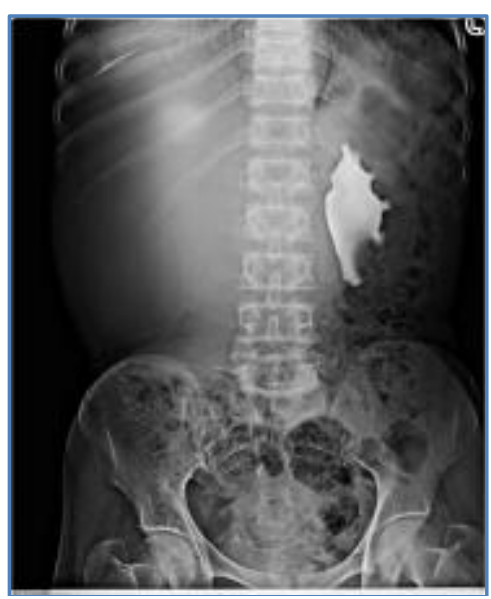

Fig. 4 


\section{CASE REPORT}

Figure 5.CECT abdomen showing a large water density lesion occupying right renal area.Hardly any renal tissue is visualized.The abdominal contents are displaced towards the left side.Left kidney shows mild dilatation of pelvicalyceal system.

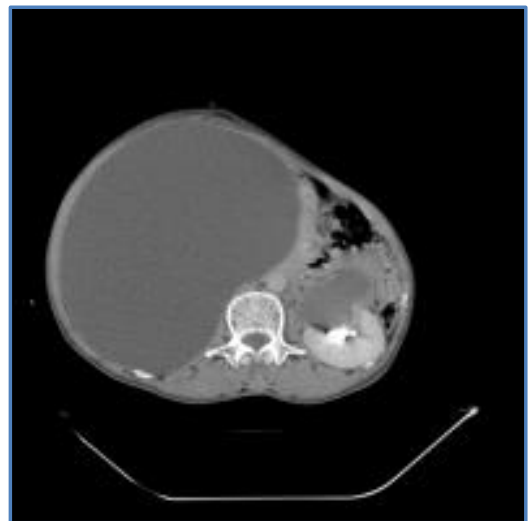

Fig. 5

\section{AUTHORS:}

1. Shiping Jain

2. Sandeep Kumar Jain

\section{PARTICULARS OF CONTRIBUTORS:}

1. Assistant Professor, Department of Radiodiagnosis, Bundelkhand Medical College, Sagar, M. P.

2. Senior Resident, Department of General Medicine, Bundelkhand Medical College, Sagar, M. P.

\section{NAME ADDRESS EMAIL ID OF THE} CORRESPONDING AUTHOR:

Dr. Shiping Jain, B-4 Block, Flat 9,

Campus of Bundel Khand Medical College, Sagar, M. P.

Email: shipingjain@yahoo.in

Date of Submission: 03/07/2014. Date of Peer Review: 04/07/2014. Date of Acceptance: 22/07/2014. Date of Publishing: 31/07/2014. 